\title{
COMPARATIVE ANALYSIS ON STATE OF CHARGE ESTIMATION TECHNIQUE FOR A BATTERY
}

\author{
Arun \\ M.Tech, ECE Department \\ B.M.S College of Engineering, \\ Bengaluru, Karnataka, India
}

\author{
Dr D Seshachalam \\ Professor, ECE Department \\ B.M.S College of Engineering \\ Bengaluru, Karnataka, India
}

\begin{abstract}
In present day, Battery technology is a major demanding and in almost all applications useful. Electric car and various applications of Battery are so dependent. Batteries require special handling with the performance and its parameters need to be monitored to prevent situations that could result in damage or unexpected burst. This is incorporated by Battery Management System. Battery Management System consists of many blocks like Sensors, Battery pack, Control Circuit, Signal lines. To provide accurate information to the Control Circuit in management system it is essential to perform various operations like $\mathrm{SOH}$, SOP and SOL. This project report describes the research in detail, and analysis of the SOC estimation method using two techniques which CC Method and EKF Method. The proposed model depicts accurate estimation method, by comparing to give the best State-of-charge. MATLAB/Simulink software is used for simulation and analysis, results show that the approach of State-ofcharge estimation using Extended Kalman Filter considering Thevenin model, provides less error compared to Coulomb Counting Method. And the report finally concludes accurate estimation technique for Battery Parameter Estimation.
\end{abstract}

Keywords - electric vehicles (EVs), State-of-charge (SOC), State-of-health (SOH), State-of-power (SOP), State-of-life (SOL). Coulomb Counting (CC), Extended Kalman Filter (EKF).

\section{INTRODUCTION}

At present, if we notice the growth of Batteries in various fields, it is not at maximum potential compared to other fields like Processor capacity, Memory and Storage capacity. It is important to improve the Battery Technology for better efficiency. Also the estimation Techniques for accurate operation in different applications. It is not easy to estimate the accurate charge of Battery because of its nonlinear behavior of Battery Characteristics. However each technique to estimate the SOC of Battery has its own advantages as well as drawback [1] [2]. Accurate estimation for Battery state of charge is a challenging task because of aging effects and its dependency on temperature [7]. This paper is to research, compare and simulate SOC Estimation Technique using Coulomb counting method and Extended Kalman Filter
Method, Further the Analysis of the work reviews the most accurate Technique.

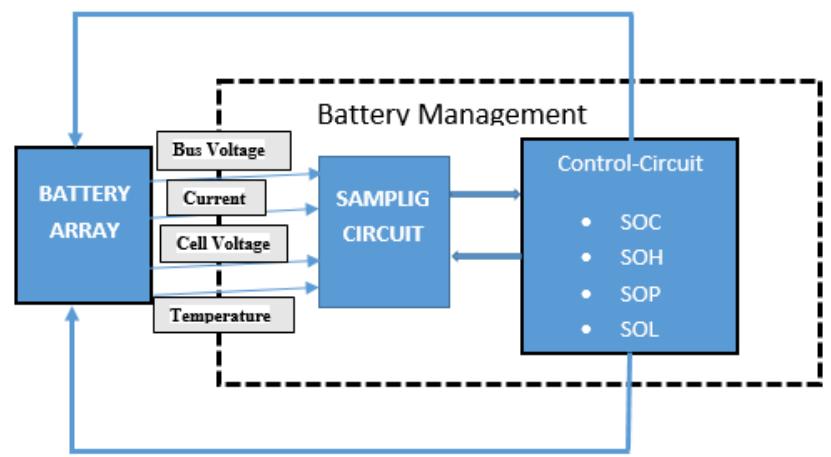

Fig. 1 Basic diagram of Battery Management System.

Battery Management System is one of prime task. To monitor and ensure safe operation of any circuit with Battery applications BMS is must. Fig 1 shows the block diagram. Battery Pack array voltage, Current and temperature various data measured from sensors and Sample Circuit analyzes and sends signal to Control Circuit to take necessary action according to the situation. Estimation of various Battery Parameter like SOC is very important in Electric Vehicles [13], Therefore it is not investigated the impact of input capacitance upon system stability for photovoltaic fed systems for other power converters. Considering the subject under review, this paper's aims are

- To research well about different SOC estimation Techniques, develop a simulation model for SOC estimation considering Thevenin model.

- Furthermore to compare Coulomb counting method and Extended Kalman Filter Method using MatLab.

- Also one of the objective of this project is to boost research in the Battery Parameter Estimation area using Industry oriented MatLab Tools.

- And to conclude the most accurate model for estimating SOC for Battery 


\section{SOC ESTIMATION METHODS}

\section{A: Electrical Equivalent Circuit Model}

Different Electrical Circuit models are considered to analyze estimate and evaluate the battery parameters for improving accuracy in different applications. The models like Rint model, RC model, Thevenin model and PNGV models and dual polarization model are used to simulate the polarization and chemical behavior of the Battery. But Thevenin model is used to depict optimal performance.

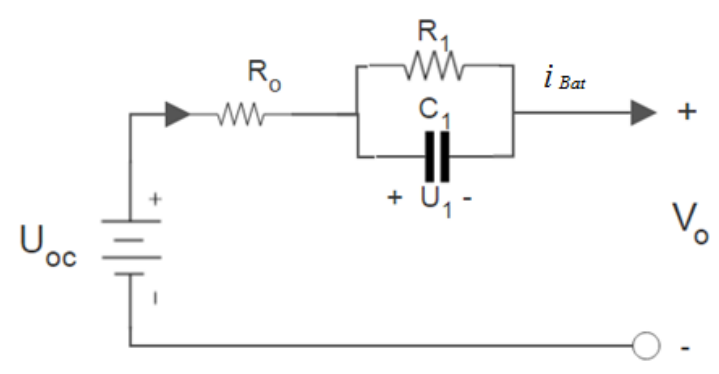

Fig.2 Equivalent Circuit of first order Thevenin model

\section{B: Coulombs Counting Method (CC)}

Coulomb Counting or Ampere-Hour method is simplest and cost effective compared to other estimation methods. But it does have problems such as an initial value must be known. And measurement error noise should be taken care of. The maximum offered capability to discharge Amperes of a battery is known and its current can be measured accurately the Coulomb Counting method easily provides the accurate estimation of the SOC.

$\operatorname{SOC}(\mathrm{t})=\operatorname{SOC}(\mathrm{t} 0)+(1 / \mathrm{Cn}) \int_{t 0}^{t 0+t}\left(I_{B a t}\right) \times 100 \%$

Equation (1) Where SOC (t0) is the initial SOC, $\mathrm{Cn}$ given capacity of Battery, and $\mathrm{I}_{\mathrm{bat}}$ is the charging/discharging current. Positive for charging and negative for discharging. $\mathrm{CC}$ has been used in most all of industry for Charge estimation At present, the $\mathrm{CC}$ method (the ampere-hour Integral method), is the most used method for SOC estimation since it is the most correct technique for short duration estimation since it is a direct method.

\section{C: EXTENDED KALMAN FILTER METHOD}

To understand the Extended Kalman filter, $\mathrm{KF}$ is an integrated method,it requires a batterymodel and a $\mathrm{s}$ tate observer to be used. Kalman filters in 1958 Rudolf Emil Kalman practiced and are developed estimation theory, for system linearity. Kalman Filter provides an accurate method for evaluating the SOC through linear filtering, the

SOC estimate of a battery remains difficult because the battery's extremely nonlinear and electrochemical behavior of the battery after usage of certain durations lead to low performance. To account this, a technique called Extended Kalman Filter (EKF) can be utilized for estimating SOC. The Extended Kalman Filter is iteration based method for nonlinear systems.

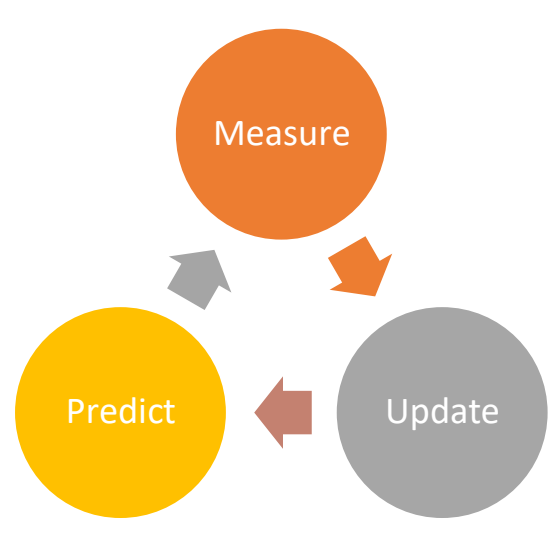

Fig.3 Kalman Filter Three Phases of Filter Operation

The figure (3) shown depicts the three stages of operation. System's state is estimated using model for each prediction transformation matrix is updated, an actual input data given to the state estimate estimation matrix and initialization of noise matrix is done. An actual input data given to the state estimate estimation matrix and initialization of noise matrix is done. The equation is defined as follows

$$
\begin{aligned}
& X_{k+1}=f\left(X_{k}, U_{k}\right)+W_{k} \\
& Y_{k}=g\left(X_{k}, U_{k}\right)+V_{k}
\end{aligned}
$$

Equation (2) shown in is processing equation, where the $\mathrm{X}_{\mathrm{k}+1}$ is state vector which consist SOC, $\mathrm{u}_{\mathrm{k}}$ is system control input, $\left(\mathrm{X}_{\mathrm{k}} \mathrm{U}_{\mathrm{k}}\right)$ is system state transition function. And $\mathrm{Y}_{\mathrm{k}}(3)$ is the output function with noise.

CC method of estimating SOC is basic and the simplest among the other. The Ampere Hour method or the CC method is implemented using the standard equation with the basic elements from Simulink Library in Matlab. The model shown in figure consists of Two Blocks CC model it is the standard equation implemented and the Battery Model is the $1^{\text {st }}$ order Thevenin equivelent model. Open circuit voltage computed over Controlled voltage source is fed to the model with $R_{o}$ connected in series with the $R C$ pair and to the terminal as controlled current source. The terminal side is connected with the controlled current source as to inject negative current equivalent to the drain current from the model when connected load. This negative current is measured with current measurement block and connected to the CC model as shown in figure (4). 


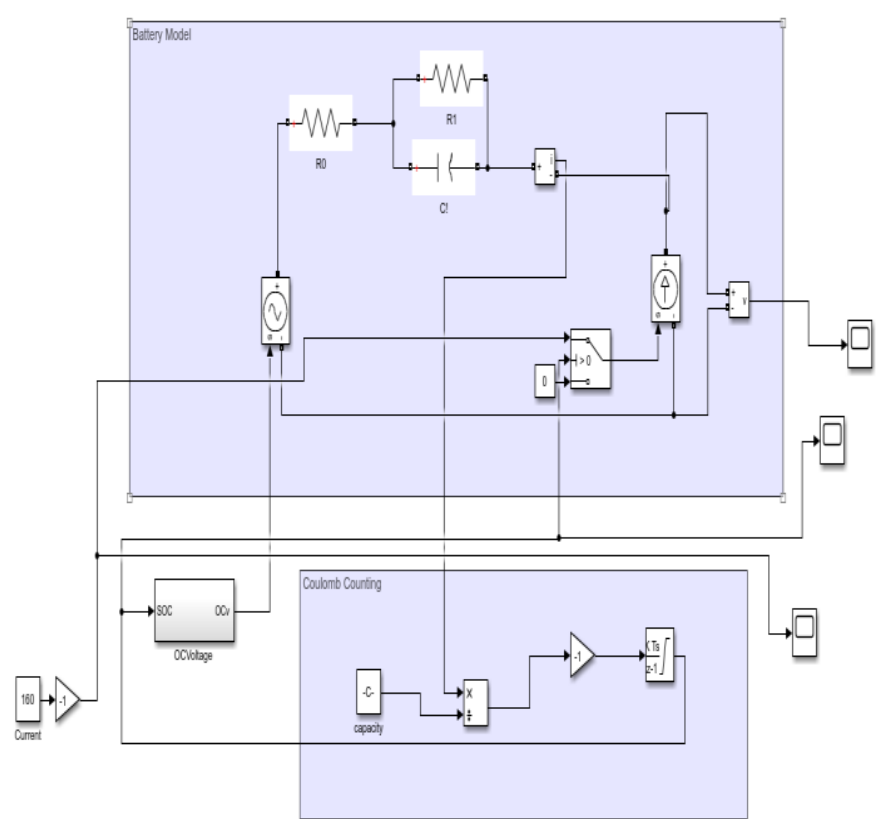

Fig.4 Simulink Block Diagram of Thevenin's Model

The CC model is Amperes measured over the time and with respect to the total capacity of the battery calculated in percentage. And integrated over the time interval, here the $\mathrm{I}_{\mathrm{Bat}}$ is negative because it is characterized the discharge. Which is following the standard equation with initial condition as hundred percent. And the gain block shows the negative current that is discharge current. In order to incorporate EKF with the input data of Discharge Current linearized model with matrices the computation is done. The equation (2) and (3) of EKF is implemented with discretized state space equations of equivalent Thevenin Circuit model. To implement EKF considered equivalent circuit model as Thevenin Circuit figure (2) transition function and discharge Current data of dynamic stress test for Battery is considered and described w.r.t matrices A, B, C and D and to apply EKF. And MatLab Code is written.

$$
\begin{aligned}
& f\left(X_{k}, U_{k}\right)=A_{k} X_{k}+B_{k} W_{k} \\
& g\left(X_{k}, U_{k}\right)=C_{k} X_{k}+D_{k} V_{k}
\end{aligned}
$$

Refer The Circuit shown in Figure (2)

$$
\begin{aligned}
& \frac{d U_{1}}{d t}=\left\{i_{\text {Bat }}\left(\frac{1}{C_{1}}\right)-\frac{U_{1}}{\left(R_{1} C_{1}\right)}\right\} \\
& V_{o}=\left\{V_{i}-U_{1}-R_{o} \times i_{\text {Bat }}\right\}
\end{aligned}
$$

$$
\begin{aligned}
& \mathrm{A}=\left[\begin{array}{ccc}
\frac{-1}{R_{1} C_{1}} & 0 & 0 \\
0 & \frac{-1}{R_{1} C_{1}} & 0 \\
0 & 0 & 0
\end{array}\right] \\
& \mathrm{B}=\left[\begin{array}{cc}
\frac{1}{C_{1}} \\
\frac{1}{C_{1}} \\
\frac{-\eta}{Q}
\end{array}\right] \\
& \mathrm{C}=\left[\begin{array}{ll}
-1 & -1 \\
\mathrm{~V} & =V_{i}
\end{array}\right] \\
& \mathrm{D}=R_{o}
\end{aligned}
$$

In the form of Modelling as:

$$
\begin{aligned}
& {\left[\begin{array}{c}
\frac{d U_{1}}{d t} \\
\frac{d S O C}{d t}
\end{array}\right]=\left[\begin{array}{cc}
\frac{-1}{R_{1} C_{1}} & 0 \\
0 & \frac{-1}{R_{1} C_{1}}
\end{array}\right]\left[\begin{array}{c}
U_{1} \\
S O C
\end{array}\right]+\left[\begin{array}{c}
\frac{1}{C_{1}} \\
\frac{-\eta}{Q}
\end{array}\right]\left[i_{B a t}\right]} \\
& {\left[U_{o c}\right]=\left[\begin{array}{ll}
-1 & V_{i}
\end{array}\right]\left[\begin{array}{c}
U_{1} \\
S O C
\end{array}\right]+R_{O} \quad\left[i_{B a t}\right]}
\end{aligned}
$$

\section{Simulation Results}

It is well known that iteration based estimation of parameter always yield more accuracy, in the Figure (5) shows the SOC estimated Curve of CC and EKF method. The upper brown color curve data shows the $\mathrm{CC}$ based and the lower cyan color data EKF based. It is noticed that the Extended Kalman Filter is more accurate because, it accounts the correction at every iteration till its meats the given threshold with present and previous state. And the new gain value is accounted for posterior steps.

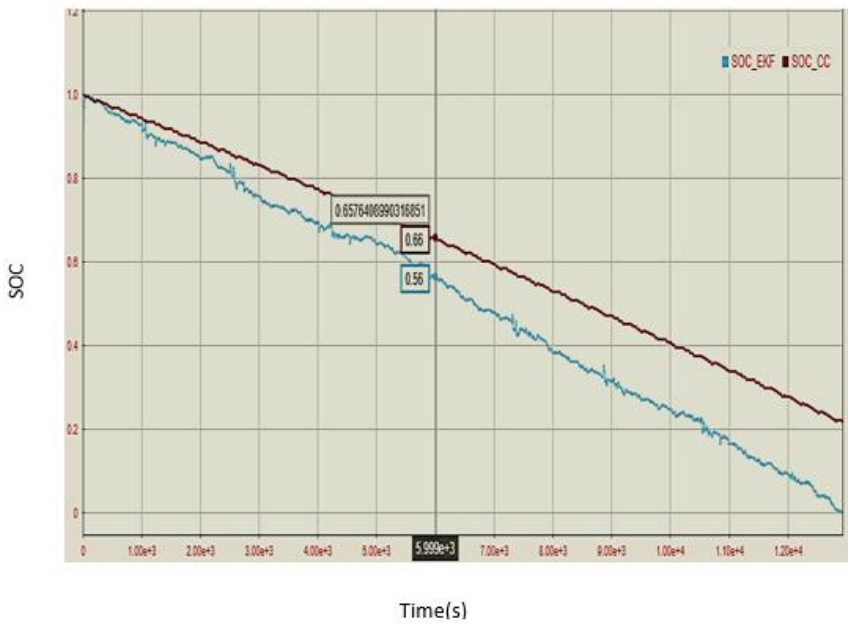

Fig.5: SOC estimation Curve of CC and EKF method 


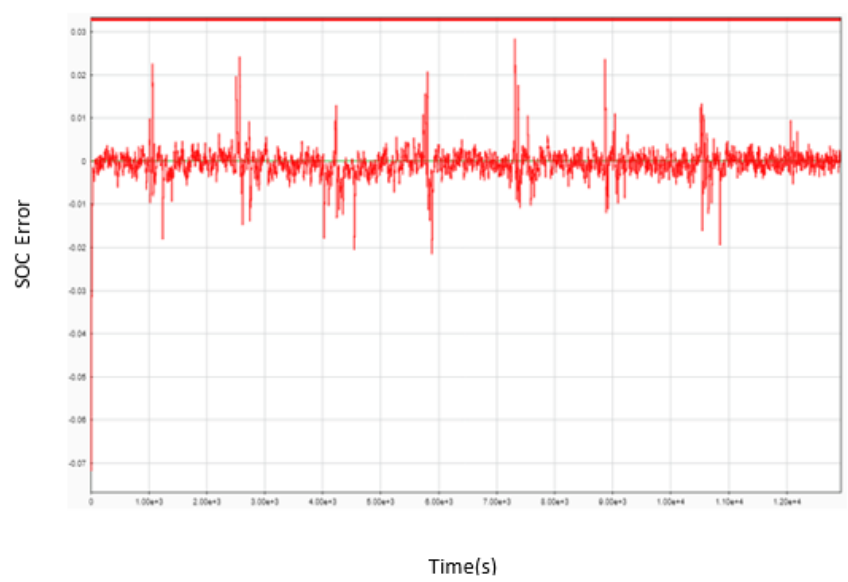

Fig.6 SOC estimation Error of CC and EKF method

\section{CONClusions}

In this paper, proposed system efficiently estimates the SOC. The Extended Kalman Filter is modelled in Simulink and Compared with the traditional $\mathrm{CC}$ method. The maximum SOC error of $3 \%$ is determined and an average error of $2 \%$ with Extended Kalman Filter Figure (6) and The data resulted by EKF model is found to be more accurate compared to the Coulombs Counting Method. Error in measuring data, change in battery temperature and accurate noise sensor errors was not taken into consideration, although the Change in discharge current is considered with dynamic stress test data. An improvement of the model with more precision can be done if considered. Reduction of error in estimating the State of Charge and meeting real time values is more demanding at the same time there is trade of with cost and complexity.

\section{FutURE SCOPE}

This is an investigation for SOC estimation design, simple and cost effective with accuracy, to estimate SOC and real time Battery Parameters considering all Battery behavior and Chemical effects is very challenge. The Battery Technology which is improving bit by bit recently, with recent $\mathrm{Li}$ Batteries of enormously developed and making it compact. There are different filter based SOC estimation of Battery but comprising reliable and cost effective and less complexity is important.

\section{REFERENCES}

[1] Juan Pablo Rivera-Barrera, Nicolás Muñoz-Galeano and Henry Omar Sarmiento-Maldonado,2017"SOC Estimation for Lithium-ion Batteries: Review and Futur Challenges", MDPI. (Molecular Diversity Preservation International).
[2] Rui xiong jiayi cao, quanqing yu, , hongwen he, and fengchun sun, 2018 "Critical Review on the Battery State of Charge Estimation Methods for Electric Vehicles" special section on battery energy storage and management systems, IEEE Access.

[3] Chen Kun, Mao Zhiwei, Lai Yuehua, Jiang Zhinong, Zhang Jinjie , 2018 " Lithium-ion battery state of charge estimation based on dynamic neural network and Kalman filter", IEEE International Conference on Prognostics and Health Management (ICPHM).

[4]Xuanju Danga, Li Yana, Kai Xua, Xiru Wua, Hui Jianga, Hanxu Sunb, 2015 "Open-Circuit Voltage-Based State of Charge Estimation of Lithium-ion Battery Using Dual Neural Network Fusion Battery Model”.

[5] Li Haoran, Li Liangdong, Zheng Xiaoyin , Zheng Xiaoyin 2018 "Lithium Battery SOC Estimation Based on Extended Kalman Filtering Algorithm", IEEE 4th International Conference on Control Science and Systems Engineering.

[6] Jihen Loukil, Ferdaous Masmoudi, and Nabil Derbel, 2017 "State of charge estimation of lead acid battery using a kalman filter", 14th International Multi-Conference on Systems, Signals \& Devices (SSD).

[7] Giulio Cossu, Wajahat Ali, Raffaele Corsini and Ernesto Ciaramella, , march 2017 "Estimation of State of Charge, Unknown Nonlinearities, and State of Health of a LithiumIon Battery Based on a Comprehensive Unobservable mode", IEEE transactions on industrial electronics, vol. 61, no. 3.

[8] Gicham Chaoui, and Hamid Gualous , march 2017 "Adaptive State of Charge Estimation of Lithium-Ion Batteries with Parameter and Thermal Uncertainties", IEEE transactions on control systems technology, vol. 25, no. 2. [9] Amir Vasebi , Maral Partovibakhsh and S. Mohammad Taghi Bathaeer, April 2007, "A novel combined battery model for state-of-charge estimation in lead-acid batteries based on extended Kalman filter for hybrid electric vehicle applications", Elsevier Journal of Power Sources 174.

[10]Feng Guang. , 2013"Modeling and simulation of SOC estimation of Li-ion battery based on EKF [D]". Wuhan: Wuhan University of Technology.

[11] Zhang, Z.L Cheng, X.; Lu, Z.Y. Gu, D.J., 2017 "SOC estimation of lithium-ion batteries with AEKF and wavelet transform matrix", IEEE Transactions on Power Electronics. [12] Ling Liu Hao, 2010 Research on SOC estimation method of lithium ion battery for electric vehicle based on EKF. Beijing: Beijing Jiaotong University.

[13] GregoryL Plett, 2004, Extended Kalman filtering for battery management systems of LiPB-based HEV battery packs Part1. Background [J]. Journal of Power Sources. 